\title{
Approximation by a kind of complex modified $q$-Durrmeyer type operators in compact disks
}

Mei-Ying Ren ${ }^{1 *}$ and Xiao-Ming Zeng ${ }^{2 *}$

"Correspondence:

npmeiyingr@163.com;

xmzeng@xmu.edu.cn

${ }^{1}$ Department of Mathematics and

Computer Science, Wuyi University,

Wuyishan, 354300, China

${ }^{2}$ Department of Mathematics,

Xiamen University, Xiamen, 361005, China

\begin{abstract}
In this paper, in order to make the convergence faster to a function being approximated, we introduce a kind of complex modified q-Durrmeyer type operators which can reproduce constant and linear functions. We study the approximation properties of these operators. We obtain the order of simultaneous approximation and a Voronovskaja-type result with a quantitative estimate for these complex modified q-Durrmeyer type operators attached to analytic functions on compact disks. More important, our results show the overconvergence phenomenon for these complex operators.
\end{abstract}

MSC: $30 \mathrm{E} 10 ; 41 \mathrm{~A} 25$

Keywords: complex modified q-Durrmeyer type operators; simultaneous approximation; Voronovskaja-type result; overconvergence; $q$-calculus

\section{Introduction}

Let $q>0$, for each nonnegative integer $k$, the $q$-integer $[k]_{q}$ and the $q$-factorial $[k]_{q}$ ! are defined by

$$
[k]_{q}:= \begin{cases}\left(1-q^{k}\right) /(1-q), & q \neq 1, \\ k, & q=1\end{cases}
$$

and

$$
[k]_{q} !:= \begin{cases}{[k]_{q}[k-1]_{q} \cdots[1]_{q},} & k \geq 1, \\ 1, & k=0,\end{cases}
$$

respectively.

Then for $q>0$ and integers $n, k, n \geq k \geq 0$, we have

$$
[k+1]_{q}=1+q[k]_{q} \text { and }[k]_{q}+q^{k}[n-k]_{q}=[n]_{q} .
$$

For the integers $n, k, n \geq k \geq 0$, the $q$-binomial coefficient is defined by

$$
\left[\begin{array}{l}
n \\
k
\end{array}\right]_{q}:=\frac{[n]_{q} !}{[k]_{q} ![n-k]_{q} !} .
$$

○ 2012 Ren and Zeng; licensee Springer. This is an Open Access article distributed under the terms of the Creative Commons Attribution License (http://creativecommons.org/licenses/by/2.0), which permits unrestricted use, distribution, and reproduction in any medium, provided the original work is properly cited. 
Let $q>0, q \neq 1$, we can define the derivative $D_{q} f$ of functions $f$ in the $q$-calculus by

$$
D_{q} f(x)= \begin{cases}\frac{f(q x)-f(x)}{(q-1) x}, & x \neq 0, \\ f^{\prime}(0), & x=0 .\end{cases}
$$

Let $a>0$, the $q$-Jackson integral in the interval $[0, a]$ is defined as

$$
\int_{0}^{a} f(t) d_{q} t=(1-q) a \sum_{j=0}^{\infty} f\left(a q^{j}\right) q^{j}, \quad 0<q<1
$$

The $q$-analogue of the Beta function is defined as

$$
B_{q}(m, n)=\int_{0}^{1} t^{m-1}(1-q t)_{q}^{n-1} d_{q} t, \quad m, n>0,
$$

where

$$
(a-b)_{q}^{n}=\prod_{j=0}^{n-1}\left(a-q^{j} b\right)
$$

Also, it is known that

$$
B_{q}(m, n)=\frac{[m-1]_{q} ![n-1]_{q} !}{[m+n-1]_{q} !} .
$$

All of the previous concepts can be found in $[1,2]$.

In 1997 Philips [3] firstly introduced and studied $q$ analogue of Bernstein polynomials. After this, the applications of $q$-calculus in the approximation theory became one of the main areas of research; many authors studied new classes of $q$-generalized operators (for instance, see [4-11]). Very recently Gupta and Wang [12] introduced and studied the following $q$-Durrmeyer operators for $0<q<1$ :

$$
T_{n, q}(f ; x)=[n+1]_{q} \sum_{k=1}^{n} q^{1-k} p_{n, k}(q ; x) \int_{0}^{1} p_{n, k-1}(q ; q t) f(t) d_{q} t+f(0) p_{n, 0}(q ; x),
$$

where $x \in[0,1], n=1,2, \ldots, 0<q<1$ and

$$
p_{n, k}(q ; x):=\left[\begin{array}{l}
n \\
k
\end{array}\right]_{q} x^{k} \prod_{s=0}^{n-k-1}\left(1-q^{s} x\right)=\left[\begin{array}{l}
n \\
k
\end{array}\right]_{q} x^{k}(1-x)_{q}^{n-k}
$$

Agarwal and Gupta [13] have extended the operators which were given by (1.1) to a complex space and have studied the approximation properties of these complex operators. They have obtained the order of approximation and a Voronovskaja-type result with a quantitative estimate for these complex operators attached to analytic functions on compact disks.

The moments of the operators $T_{n, q}(f ; x)$ were obtained as follows (see [12]): 
Remark 1 Denote $e_{k}(x)=x^{k}, k=0,1,2$. For $0<q<1, x \in[0,1], n \in \mathbb{N}$, we have

$$
\begin{aligned}
& T_{n, q}\left(e_{0} ; x\right)=1, \quad T_{n, q}\left(e_{1} ; x\right)=\frac{[n]_{q} x}{[n+2]_{q}}, \\
& T_{n, q}\left(e_{2} ; x\right)=x \frac{(1+q)[n]_{q}}{[n+3]_{q}[n+2]_{q}}+x^{2} \frac{q[n]_{q}\left([n]_{q}-1\right)}{[n+3]_{q}[n+2]_{q}} .
\end{aligned}
$$

It can be observed from the above remark that the operators $T_{n, q}(f ; x)$ reproduce only a constant function. To make the convergence faster, we modify these operators so that they reproduce constant as well as linear functions. For this reason, we change the scale of reference by replacing the term $x$ by $\frac{[n+2]_{q} x}{[n]_{q}}$, in the definition of $T_{n, q}(f ; x)$ given by (1.1). Using the restriction $x \in\left[0, \frac{1}{[3]_{q}}\right]$, we have the following positive linear operators:

$$
R_{n, q}(f ; x)=[n+1]_{q} \sum_{k=1}^{n} q^{1-k} t_{n, k}(q ; x) \int_{0}^{1} p_{n, k-1}(q ; q t) f(t) d_{q} t+f(0) t_{n, 0}(q ; x),
$$

where $x \in\left[0, \frac{1}{[3]_{q}}\right], n \in \mathbb{N}, 0<q<1$, the term $p_{n, k}(q ; x)$ is given in $(1.1)$ and

$$
t_{n, k}(q ; x):=\left[\begin{array}{l}
n \\
k
\end{array}\right]_{q}\left(\frac{[n+2]_{q}}{[n]_{q}} x\right)^{k}\left(1-\frac{[n+2]_{q}}{[n]_{q}} x\right)_{q}^{n-k}
$$

By simple computation, we get the moments of the operators $R_{n, q}(f ; x)$.

Remark 2 Denoting $e_{k}(x)=x^{k}, k=0,1,2$, for $0<q<1, x \in\left[0, \frac{1}{[3]_{q}}\right], n \in \mathbb{N}$, we have

$$
R_{n, q}\left(e_{0} ; x\right)=1, \quad R_{n, q}\left(e_{1} ; x\right)=x, \quad R_{n, q}\left(e_{2} ; x\right)=x \frac{1+q}{[n+3]_{q}}+x^{2} \frac{q^{2}[n-1]_{q}[n+2]_{q}}{[n+3]_{q}[n]_{q}}
$$

The aim of the present article is to obtain approximation results for the complex extension of the $q$-Bernstein-Durrmeyer type modified operator (1.2) defined by

$$
M_{n, q}(f ; z)=[n+1]_{q} \sum_{k=1}^{n} q^{1-k} t_{n, k}(q ; z) \int_{0}^{1} p_{n, k-1}(q ; q t) f(t) d_{q} t+f(0) t_{n, 0}(q ; z),
$$

where $z \in \mathbb{C}, n=1,2, \ldots, 0<q<1$ and $t_{n, k}(q ; z):=\left[\begin{array}{l}n \\ k\end{array}\right]_{q}\left(\frac{[n+2]_{q}}{[n]_{q}} z\right)^{k}\left(1-\frac{[n+2]_{q}}{[n]_{q}} z\right)_{q}^{n-k}, p_{n, k}(q ; z):=$ $\left[\begin{array}{l}n \\ k\end{array}\right]_{q} z^{k}(1-z)_{q}^{n-k}$

\section{Auxiliary results}

In the sequel, we shall need the following auxiliary results.

Lemma 1 Let $0<q<1, m \in \mathbb{N}$. We have $M_{n, q}\left(t^{m} ; z\right)$ is a polynomial of degree $\leq \min (m, n)$ and

$$
M_{n, q}\left(t^{m} ; z\right)=\frac{[n+1]_{q} !}{[n+m+1]_{q} !} \sum_{s=1}^{m} c_{s}(m)[n]_{q}^{s} B_{n, q}\left(t^{s} ; z\right)
$$


where $c_{s}(m) \geq 0$ are constants depending on $m$ and $q$ and

$$
B_{n, q}(f ; z)=\sum_{k=0}^{n} t_{n, k}(q ; z) f\left([k]_{q} /[n]_{q}\right)
$$

Proof By the definition of $q$-Beta function, we have

$$
\begin{aligned}
M_{n, q}\left(t^{m} ; z\right) & =[n+1]_{q} \sum_{k=1}^{n} q^{1-k} t_{n, k}(q ; z) \int_{0}^{1} p_{n, k-1}(q ; q t) t^{m} d_{q} t \\
& =\frac{[n+1]_{q} !}{[n+m+1]_{q} !} \sum_{k=1}^{n} t_{n, k}(q ; z) \frac{[k+m-1]_{q} !}{[k-1]_{q} !} .
\end{aligned}
$$

Considering the definition of the $B_{n, q}(f ; z)$, for any $m \in \mathbb{N}$, applying the principle of mathematical induction, we immediately obtain the desired conclusion.

Lemma 2 Let $0<q<1$. For all $m, n \in \mathbb{N}$, we can get the inequality

$$
\frac{[n+1]_{q} !}{[n+m+1]_{q} !} \sum_{s=1}^{m} c_{s}(m)[n]_{q}^{s} \leq 1
$$

Proof By Lemma 1, we have

$$
\begin{aligned}
M_{n, q}\left(t^{m} ; \frac{[n]_{q}}{[n+2]_{q}}\right) & =\frac{[n+1]_{q} !}{[n+m+1]_{q} !} \sum_{s=1}^{m} c_{s}(m)[n]_{q}^{s} B_{n, q}\left(t^{s} ; \frac{[n]_{q}}{[n+2]_{q}}\right) \\
& =\frac{[n+1]_{q} !}{[n+m+1]_{q} !} \sum_{s=1}^{m} c_{s}(m)[n]_{q}^{s} .
\end{aligned}
$$

On the other hand, we have $t_{n, k}\left(q ; \frac{[n]_{q}}{[n+2]_{q}}\right)=0, k=0,1,2, \ldots, n-1$, also $t_{n, n}(q ; z)=\left(\frac{[n+2]_{q}}{[n]_{q}} z\right)^{n}$ and $t_{n, n}\left(q ; \frac{[n]_{q}}{[n+2]_{q}}\right)=1$. So, by formula (1.3) and using the above values, we have

$$
\begin{aligned}
M_{n, q}\left(t^{m} ; \frac{[n]_{q}}{[n+2]_{q}}\right) & =[n+1]_{q} t_{n, n}\left(q ; \frac{[n]_{q}}{[n+2]_{q}}\right) q^{1-n} \int_{0}^{1} p_{n, n-1}(q ; q t) t^{m} d_{q} t \\
& =\frac{[n]_{q}[n+1]_{q}}{[n+m]_{q}[n+m+1]_{q}} \leq 1,
\end{aligned}
$$

which implies that we get the desired conclusion.

Corollary 1 Denote $e_{m}(t)=t^{m}$, let $r \geq 1$ and $0<q<1$. Then for all $m \in \mathbb{N} \cup\{0\}$ and $|z| \leq r$, we have $\left|M_{n, q}\left(e_{m} ; z\right)\right| \leq\left([3]_{q} r\right)^{m}$.

Lemma 3 Let $0<q<1, e_{m}(t)=t^{m}, m \in \mathbb{N} \cup\{0\}$ and $z \in \mathbb{C}$, we have

$$
\begin{aligned}
M_{n, q}\left(e_{m+1} ; z\right)= & \frac{q^{m} z\left([n]_{q}-[n+2]_{q} z\right)}{[n]_{q}[m+n+2]_{q}} D_{q} M_{n, q}\left(e_{m} ; z\right) \\
& +\frac{[m]_{q}+q^{m}[n+2]_{q} z}{[m+n+2]_{q}} M_{n, q}\left(e_{m} ; z\right) .
\end{aligned}
$$


Ken and Zens Journal of Inequalities and Applications 2012, 2012:212

Page 5 of 14

Proof By Lemma 1, we have $M_{n, q}\left(e_{0} ; z\right)=1$ and $M_{n, q}\left(e_{1} ; z\right)=z$, therefore, this result is established for $m=0$. Now, let $m \in \mathbb{N}$, in view of $D_{q}(f(x) g(x))=g(x) D_{q} f(x)+f(q x) D_{q} g(x)$ and $D_{q}(a+b x)_{q}^{n}=[n]_{q} b(a+b q x)_{q}^{n-1}$, by simple calculation, we obtain

$$
\begin{aligned}
& z\left(1-\frac{[n+2]_{q}}{[n]_{q}} z\right) D_{q}\left(t_{n, k}(q ; z)\right)=\left([k]_{q}-[n+2]_{q} z\right) t_{n, k}(q ; z), \\
& z(1-z) D_{q}\left(p_{n, k}(q ; z)\right)=\left([k]_{q}-[n]_{q} z\right) p_{n, k}(q ; z) .
\end{aligned}
$$

For $u=u(t)=\alpha t(\alpha$ is a constant $)$, since $D_{q} f(u(t))=D_{q} f(u) \cdot D_{q} u(t)$, therefore, we have

$$
\begin{aligned}
& t(1-q t) D_{q}\left(p_{n, k}(q ; q t)\right)=p_{n, k}(q ; q t)\left([k]_{q}-[n]_{q} q t\right) \\
& t(1-q t) D_{q}\left(p_{n, k-1}(q ; q t)\right)=p_{n, k-1}(q ; q t)\left([k-1]_{q}-[n]_{q} q t\right) .
\end{aligned}
$$

It follows that

$$
\begin{aligned}
z(1- & \left.\frac{[n+2]_{q}}{[n]_{q}} z\right) D_{q}\left(M_{n, q}\left(e_{m} ; z\right)\right) \\
= & {[n+1]_{q} \sum_{k=1}^{n} q^{1-k}\left([k]_{q}-[n+2]_{q} z\right) t_{n, k}(q ; z) \int_{0}^{1} p_{n, k-1}(q ; q t) t^{m} d_{q} t } \\
= & {[n+1]_{q} \sum_{k=1}^{n} q^{1-k} t_{n, k}(q ; z) \int_{0}^{1}\left(1+q[k-1]_{q}-[n]_{q} q^{2} t+[n]_{q} q^{2} t\right) p_{n, k-1}(q ; q t) t^{m} d_{q} t } \\
& -z[n+2]_{q}[n+1]_{q} \sum_{k=1}^{n} q^{1-k} t_{n, k}(q ; z) \int_{0}^{1} p_{n, k-1}(q ; q t) t^{m} d_{q} t \\
= & q[n+1]_{q} \sum_{k=1}^{n} q^{1-k} t_{n, k}(q ; z) \int_{0}^{1} D_{q}\left(p_{n, k-1}(q ; q t)\right) t(1-q t) t^{m} d_{q} t \\
& +M_{n, q}\left(e_{m} ; z\right)+q^{2}[n]_{q} M_{n, q}\left(e_{m+1} ; z\right)-z[n+2]_{q} M_{n, q}\left(e_{m} ; z\right) .
\end{aligned}
$$

Letting $\delta(t)=\frac{t}{q}(1-t)\left(\frac{t}{q}\right)^{m}$, using $q$-integrate by parts, we have

$$
\int_{0}^{1} D_{q}(f(t)) \delta(q t) d_{q} t=\left.\delta(t) f(t)\right|_{0} ^{1}-\int_{0}^{1} f(t) D_{q} \delta(t) d_{q} t .
$$

So, the $q$-integral in the above formula becomes

$$
\begin{aligned}
\int_{0}^{1} & D_{q}\left(p_{n, k-1}(q ; q t)\right) t(1-q t) t^{m} d_{q} t \\
\quad= & \left.\delta(t) p_{n, k-1}(q ; q t)\right|_{0} ^{1}-\int_{0}^{1} p_{n, k-1}(q ; q t) D_{q} \delta(t) d_{q} t \\
& =-q^{-m-1} \int_{0}^{1} p_{n, k-1}(q ; q t) D_{q}\left(t^{m+1}-t^{m+2}\right) d_{q} t \\
& =-q^{-m-1}[m+1]_{q} \int_{0}^{1} p_{n, k-1}(q ; q t) t^{m} d_{q} t+q^{-m-1}[m+2]_{q} \int_{0}^{1} p_{n, k-1}(q ; q t) t^{m+1} d_{q} t
\end{aligned}
$$


Thus, we obtain

$$
\begin{aligned}
z\left(1-\frac{[n+2]_{q}}{[n]_{q}} z\right) D_{q}\left(M_{n, q}\left(e_{m} ; z\right)\right)= & -q^{-m}[m+1]_{q} M_{n, q}\left(e_{m} ; z\right) \\
& +q^{-m}[m+2]_{q} M_{n, q}\left(e_{m+1} ; z\right)+M_{n, q}\left(e_{m} ; z\right) \\
& +q^{2}[n]_{q} M_{n, q}\left(e_{m+1} ; z\right)-z[n+2]_{q} M_{n, q}\left(e_{m} ; z\right) .
\end{aligned}
$$

In view of $[m+2]_{q}+q^{m+2}[n]_{q}=[m+n+2]_{q}$ and $[m+1]_{q}=[m]_{q}+q^{m}$, by simple calculation, we can get the recurrence in the statement.

Lemma 4 Denote $S_{n, m}(q ; z)=M_{n, q}\left(e_{m} ; z\right)-z^{m}$. Let $0<q<1, e_{m}(t)=t^{m}$, for all $m \in \mathbb{N} \cup\{0\}$ and $z \in \mathbb{C}$, we have

$$
\begin{aligned}
S_{n, m}(q ; z)= & \frac{q^{m-1} z\left([n]_{q}-[n+2]_{q} z\right)}{[n]_{q}[m+n+1]_{q}} D_{q} M_{n, q}\left(e_{m-1} ; z\right) \\
& +\frac{[m-1]_{q}+z q^{m-1}[n+2]_{q}}{[m+n+1]_{q}} S_{n, m-1}(q ; z)+\frac{[m-1]_{q}(1-z) z^{m-1}}{[m+n+1]_{q}} .
\end{aligned}
$$

Proof Using formula (2.1), by simple calculation, we can easily get the recurrence (2.2), the proof is omitted here.

Lemma 5 If $P_{m}(z)$ is a polynomial of degree $m$, for all $|z| \leq r$, we have

$$
\left|D_{q} P_{m}(z)\right| \leq\left\|P_{m}^{\prime}\right\|_{r} \leq \frac{m}{r}\left\|P_{m}\right\|_{r}
$$

where $\left\|P_{m}\right\|_{r}=\max \left\{\left|P_{m}(z)\right| ;|z| \leq r\right\}$.

Proof The proof is easy by using the Bernstein inequality and the complex mean value theorem, the proof is omitted here.

Let $e_{m}(t)=t^{m}, m \in \mathbb{N}$. By Lemma 1 , for all $|z| \leq r$, we have

$$
\left|D_{q} M_{n, q}\left(e_{m-1} ; z\right)\right| \leq \frac{m-1}{r}\left\|M_{n, q}\left(e_{m-1} ; \cdot\right)\right\|_{r} .
$$

\section{Main results}

The first main result is expressed by the following upper estimates.

Theorem 1 Let $0<q<1, R>3, D_{R}=\{z \in \mathbb{C}:|z|<R\}$. Suppose that $f: D_{R} \rightarrow \mathbb{C}$ is analytic in $D_{R}$, i.e., $f(z)=\sum_{m=0}^{\infty} c_{m} z^{m}$ for all $z \in D_{R}$. Take $1 \leq r \leq \frac{R}{3}$.

(i) For all $|z| \leq r$ and $n \in \mathbb{N}$, we have

$$
\left|M_{n, q}(f ; z)-f(z)\right| \leq \frac{K_{r}(f)}{|n|_{q}},
$$

where $K_{r}(f)=(1+r) \sum_{m=2}^{\infty}\left|c_{m}\right| m(m-1)\left([3]_{q} r\right)^{m-1}<\infty$. 
(ii) (Simultaneous approximation) If $1 \leq r<r_{1}<\frac{R}{3}$ are arbitrary fixed, then for all $|z| \leq r$ and $n, p \in \mathbb{N}$, we have

$$
\left|M_{n, q}^{(p)}(f ; z)-f^{(p)}(z)\right| \leq \frac{K_{r_{1}}(f) p ! r_{1}}{[n]_{q}\left(r_{1}-r\right)^{p+1}}
$$

where $K_{r_{1}}(f)$ is defined as in (i) above.

Proof Taking $e_{m}(z)=z^{m}$, by the hypothesis that $f(z)$ is analytic in $D_{R}$, i.e., $f(z)=\sum_{m=0}^{\infty} c_{m} z^{m}$ for all $z \in D_{R}$, it is easy for us to obtain $M_{n, q}(f ; z)=\sum_{m=0}^{\infty} c_{m} M_{n, q}\left(e_{m} ; z\right)$, therefore, we get

$$
\begin{aligned}
\left|M_{n, q}(f ; z)-f(z)\right| & \leq \sum_{m=0}^{\infty}\left|c_{m}\right| \cdot\left|M_{n, q}\left(e_{m} ; z\right)-e_{m}(z)\right| \\
& =\sum_{m=2}^{\infty}\left|c_{m}\right| \cdot\left|M_{n, q}\left(e_{m} ; z\right)-e_{m}(z)\right|,
\end{aligned}
$$

as $M_{n, q}\left(e_{0} ; z\right)=1, M_{n, q}\left(e_{1} ; z\right)=z$.

(i) By Lemma 4, Lemma 5 and Corollary 1 , for all $m \in \mathbb{N}$, we get

$$
\begin{aligned}
\left|M_{n, q}\left(e_{m} ; z\right)-e_{m}(z)\right|= & \left|S_{n, m}(q ; z)\right| \leq \frac{r(1+r)}{[n]_{q}} \cdot \frac{m-1}{r}\left\|M_{n, q}\left(e_{m-1 ;}\right)\right\|_{r} \\
& +r\left|S_{n, m-1}(q ; z)\right|+\frac{m-1}{[n]_{q}}(1+r) r^{m-1} \\
\leq & \frac{2(m-1)}{[n]_{q}}(1+r)\left([3]_{q} r\right)^{m-1}+r\left|S_{n, m-1}(q ; z)\right| .
\end{aligned}
$$

By writing the last inequality, for $m=2,3, \ldots$, we easily obtain

$$
\left|M_{n, q}\left(e_{m} ; z\right)-e_{m}(z)\right| \leq \frac{1+r}{[n]_{q}} m(m-1)\left([3]_{q} r\right)^{m-1} .
$$

In conclusion, it follows that

$$
\left|M_{n, q}(f ; z)-f(z)\right| \leq \frac{1+r}{[n]_{q}} \sum_{m=2}^{\infty}\left|c_{m}\right| m(m-1)\left([3]_{q} r\right)^{m-1}
$$

By the hypothesis on $f$, we have $f^{(2)}(z)=\sum_{m=2}^{\infty} c_{m} m(m-1) z^{m-2}$, and the series is absolutely convergent in $|z| \leq[3]_{q} r$, so we get $\sum_{m=2}^{\infty}\left|c_{m}\right| m(m-1)\left([3]_{q} r\right)^{m-2}<\infty$, that is $K_{r}(f)=(1+r) \sum_{m=2}^{\infty}\left|c_{m}\right| m(m-1)\left([3]_{q} r\right)^{m-1}<\infty$.

(ii) Denoting by $\Gamma$ the circle of radius $r_{1}>r$ and center 0 , since for any $|z| \leq r$ and $v \in \Gamma$ we have $|v-z| \geq r_{1}-r$, by the Cauchy's formulas it follows that for all $|z| \leq r$ and $n, p \in \mathbb{N}$, we have

$$
\begin{aligned}
\left|M_{n, q}^{(p)}(f ; z)-f^{p}(z)\right| & =\frac{p !}{2 \pi}\left|\int_{\Gamma} \frac{M_{n, q}(f ; v)-f(v)}{(v-z)^{p+1}} d v\right| \\
& \leq \frac{K_{r_{1}}(f)}{[n]_{q}} \frac{p !}{2 \pi} \frac{2 \pi r_{1}}{\left(r_{1}-r\right)^{p+1}}=\frac{K_{r_{1}}(f)}{[n]_{q}} \cdot \frac{p ! r_{1}}{\left(r_{1}-r\right)^{p+1}},
\end{aligned}
$$

which proves the theorem. 
Remark 3 Let $0<q<1$ be fixed. Since we have $\frac{1}{[n]_{q}} \rightarrow 1-q$ as $n \rightarrow \infty$, by passing to limit with $n \rightarrow \infty$ in the estimates in Theorem 1, we do not obtain the convergence of $M_{n, q}^{(p)}(f ; z)$ to $f^{(p)}(z), p=0,1, \ldots$. But this situation can be improved by choosing $0<q_{n}<1$ with $q_{n} \rightarrow 1$ as $n \rightarrow \infty$. Indeed, since in this case $\frac{1}{[n] q_{n}} \rightarrow 0$ as $n \rightarrow \infty$ (see Videnskii [14], formula (2.7)), from Theorem 1 we get that $M_{n, q}^{(p)}(f ; z) \rightarrow f^{(p)}(z)$, for $p=0,1, \ldots$, uniformly for $|z| \leq r$, for any $1 \leq r<r_{1}<\frac{R}{3}$.

The following Voronovskaja-type result with a quantitative estimate holds.

Theorem 2 Let $0<q<1, R>3$ and suppose that $f: D_{R} \rightarrow \mathbb{C}$ is analytic in $D_{R}=\{z \in \mathbb{C}$ : $|z|<R\}$, i.e., $f(z)=\sum_{k=0}^{\infty} c_{k} z^{k}$ for all $z \in D_{R}$. For any fixed $r \in\left[1, \frac{R}{3}\right]$ and for all $n \in \mathbb{N},|z| \leq r$, we have

$$
\left|M_{n, q}(f ; z)-f(z)-\frac{z(1-z) f^{\prime \prime}(z)}{[n]_{q}}\right| \leq \frac{M_{r}(f)}{[n]_{q}^{2}},
$$

where $M_{r}(f)=\sum_{k=2}^{\infty}\left|c_{k}\right|(k-1) F_{k, r}\left([3]_{q} r\right)^{k}<\infty$ and $F_{k, r}=(k-1)(k-2)(2 k-3)+6 k(k-1)^{2}+$ $4(k-1) k^{2}+4(k-2)(k-1)^{2}(1+r)$.

Proof Denoting $e_{k}(z)=z^{k}, k=0,1,2, \ldots$, by the hypothesis that $f(z)$ is analytic in $D_{R}$, i.e., $f(z)=\sum_{k=0}^{\infty} c_{k} z^{k}$ for all $z \in D_{R}$, we can write $M_{n, q}(f ; z)=\sum_{k=0}^{\infty} c_{k} M_{n, q}\left(e_{k} ; z\right)$, thus, for all $z \in D_{R}, n \in \mathbb{N}$, we have

$$
\begin{aligned}
& \left|M_{n, q}(f ; z)-f(z)-\frac{z(1-z) f^{\prime \prime}(z)}{[n]_{q}}\right| \\
& \quad \leq \sum_{k=2}^{\infty}\left|c_{k}\right|\left|M_{n, q}\left(e_{k} ; z\right)-e_{k}(z)-\frac{k(k-1)(1-z) z^{k-1}}{[n]_{q}}\right| .
\end{aligned}
$$

Denoting

$$
E_{k, n}(q ; z)=M_{n, q}\left(e_{k} ; z\right)-e_{k}(z)-\frac{k(k-1)(1-z) z^{k-1}}{[n]_{q}},
$$

it is obvious that $E_{k, n}(q ; z)$ is a polynomial of degree less than or equal to $k$. By simple computation and the use of Lemma 3 , for all $k \geq 2$, we can get

$$
\begin{aligned}
E_{k, n}(q ; z)= & \frac{q^{k-1} z\left([n]_{q}-[n+2]_{q} z\right)}{[n]_{q}[n+k+1]_{q}} D_{q} E_{k-1, n}(q ; z) \\
& +\frac{q^{k-1}[n+2]_{q} z+[k-1]_{q}}{[n+k+1]_{q}} E_{k-1, n}(q ; z)+G_{k, n}(q ; z),
\end{aligned}
$$

where

$$
\begin{aligned}
G_{k, n}(q ; z)= & \frac{z^{k-2}}{[n]_{q}^{2}[n+k+1]_{q}}\left\{z ^ { 2 } \left[-q^{k-1}[k-1]_{q}[n]_{q}[n+2]_{q}\right.\right. \\
& +q^{k-1}(k-1)(k-2)[n+2]_{q} \\
& +q^{k}(k-1)(k-2)[k-2]_{q}[n+2]_{q}+q^{k-1}[n+2]_{q}[n]_{q}^{2}
\end{aligned}
$$




$$
\begin{aligned}
& -q^{k-1}(k-1)(k-2)[n]_{q}[n+2]_{q}-[n+k+1]_{q}[n]_{q}^{2} \\
& \left.+k(k-1)[n+k+1]_{q}[n]_{q}\right] \\
& +z\left[q^{k-1}[k-1]_{q}[n]_{q}^{2}-q^{k-1}(k-1)(k-2)[n]_{q}-q^{k}(k-1)(k-2)[k-2]_{q}[n]_{q}\right. \\
& -q^{k-1}(k-1)(k-2)[k-2]_{q}[n+2]_{q} \\
& +[k-1]_{q}[n]_{q}^{2}-(k-1)(k-2)[k-1]_{q}[n]_{q} \\
& \left.+q^{k-1}(k-1)(k-2)[n]_{q}[n+2]_{q}-k(k-1)[n+k+1]_{q}[n]_{q}\right] \\
& \left.+\left[q^{k-1}(k-1)(k-2)[k-2]_{q}[n]_{q}+(k-1)(k-2)[k-1]_{q}[n]_{q}\right]\right\} \\
:= & \frac{z^{k-2}}{[n]_{q}^{2}[n+k+1]_{q}}\left(z^{2} A_{k, n}(q)+z B_{k, n}(q)+C_{k, n}(q)\right) .
\end{aligned}
$$

For all $k \geq 2$, we easily obtain $\left|C_{k, n}(q)\right| \leq[n]_{q}(k-1)(k-2)(2 k-3)$, it follows that

$$
\left|\frac{z^{k-2} C_{k, n}(q)}{[n]_{q}^{2}[n+k+1]_{q}}\right| \leq \frac{(k-1)(k-2)(2 k-3) r^{k}}{[n]_{q}^{2}}
$$

In view of $[n+k+1]_{q}=[k-1]_{q}+q^{k-1}[n]_{q}+q^{n+k-1}+q^{n+k}$ and $[n+2]_{q}=[n]_{q}+q^{n}+q^{n+1}$, for all $k \geq 2$, we can get

$$
\begin{aligned}
B_{k, n}(q)= & {[n]_{q}^{2}\left[q^{k-1}\left([k-1]_{q}-(k-1)\right)+\left([k-1]_{q}-q^{k-1}(k-1)\right)\right] } \\
& +[n]_{q}\left[-q^{k-1}(k-1)(k-2)\left(1+q[k-2]_{q}+[k-2]_{q}\right)-2(k-1)^{2}[k-1]_{q}\right. \\
& \left.-2 q^{n+k-1}(1+q)(k-1)\right]-q^{n+k-1}(1+q)(k-1)(k-2)[k-2]_{q} .
\end{aligned}
$$

Also, according to $[k-1]_{q}-(k-1)=(q-1) \sum_{j=0}^{k-2}[j]_{q}$ and $[k-1]_{q}-q^{k-1}(k-1)=(1-$ q) $\sum_{j=1}^{k-1}[j]_{q} q^{k-1-j}$, we have

$$
\begin{aligned}
& \left|[n]_{q}^{2}\left[q^{k-1}\left([k-1]_{q}-(k-1)\right)+\left([k-1]_{q}-q^{k-1}(k-1)\right)\right]\right| \\
& \quad=\left|[n]_{q} q^{k-1}\left(q^{n}-1\right) \sum_{j=0}^{k-2}[j]_{q}+[n]_{q}\left(1-q^{n}\right) \sum_{j=1}^{k-1}[j]_{q} q^{k-1-j}\right| \\
& \quad \leq[n]_{q}\left[(k-1)[k-2]_{q}+(k-1)[k-1]_{q}\right] .
\end{aligned}
$$

Thus, through simple calculation, we can get

$$
\left|\frac{z^{k-1} B_{k, n}(q)}{[n]_{q}^{2}[n+k+1]_{q}}\right| \leq \frac{6 k(k-1)^{2} r^{k}}{[n]_{q}^{2}} .
$$

Now, we estimate $A_{k, n}(q)$. Similar to the calculation of $B_{k, n}(q)$, for all $k \geq 2$, we have

$$
\begin{aligned}
A_{k, n}(q)= & -[n]_{q}\left[q^{k-1}\left(q^{n}-1\right) \sum_{j=0}^{k-2}[j]_{q}+\left(1-q^{n}\right) \sum_{j=1}^{k-1}[j]_{q} q^{k-1-j}\right]+[n]_{q}\left[k(k-1)[k-1]_{q}\right. \\
& \left.+q^{k-1}(k-1)(k-2)\left(1+q[k-2]_{q}\right)-q^{n+k-1}(1+q)\left([k-1]_{q}-2 k+2\right)\right] \\
& +q^{n+k-1}(1+q)(k-1)(k-2)\left(1+q[k-2]_{q}\right) .
\end{aligned}
$$


By simple calculation, it follows that

$$
\left|\frac{z^{k} A_{k, n}(q)}{[n]_{q}^{2}[n+k+1]_{q}}\right| \leq \frac{4(k-1) k^{2} r^{k}}{[n]_{q}^{2}} .
$$

Thus, for all $k \geq 2, n \in \mathbb{N}$ and $|z| \leq r$, we can obtain

$$
\left|G_{k, n}(q ; z)\right| \leq \frac{r^{k}}{[n]_{q}^{2}}\left[(k-1)(k-2)(2 k-3)+6 k(k-1)^{2}+4(k-1) k^{2}\right]:=\frac{r^{k}}{[n]_{q}^{2}} D_{k},
$$

where $D_{k}=(k-1)(k-2)(2 k-3)+6 k(k-1)^{2}+4(k-1) k^{2}$.

For all $k \geq 2, n \in \mathbb{N}$ and $|z| \leq r, 1 \leq r$, it follows

$$
\begin{aligned}
\left|E_{k, n}(q ; z)\right| \leq & \frac{r(1+r)}{[n]_{q}}\left|D_{q} E_{k-1, n}(q ; z)\right| \\
& +\frac{q^{k-1}[n+2]_{q} r+[k-1]_{q}}{[n+k+1]_{q}}\left|E_{k-1, n}(q ; z)\right|+\left|G_{k, n}(q ; z)\right| .
\end{aligned}
$$

Since $q^{k-1}[n+2]_{q} r+[k-1]_{q} \leq[n+k+1]_{q} r$, it follows

$$
\left|E_{k, n}(q ; z)\right| \leq \frac{r(1+r)}{[n]_{q}}\left|D_{q} E_{k-1, n}(q ; z)\right|+r\left|E_{k-1, n}(q ; z)\right|+\left|G_{k, n}(q ; z)\right| .
$$

Using the estimate in the proof of Theorem 1(i), we get

$$
\left|M_{n, q}\left(e_{k} ; z\right)-e_{k}(z)\right| \leq \frac{1+r}{[n]_{q}} k(k-1)\left([3]_{q} r\right)^{k-1}
$$

for all $k, n \in \mathbb{N},|z| \leq r, 1 \leq r$.

Denote $\|f\|_{r}=\max \{|f(z)| ;|z| \leq r\}$, by Lemma 5, we have

$$
\begin{aligned}
& \left|D_{q} E_{k-1, n}(q ; z)\right| \\
& \quad \leq \frac{k-1}{r}\left\|E_{k-1, n}\right\|_{r} \\
& \quad \leq \frac{k-1}{r}\left[\left\|M_{n, q}\left(e_{k-1} ; \cdot\right)-e_{k-1}\right\|_{r}+\left\|\frac{(k-1)(k-2)\left(1-e_{1}\right) e_{k-2}}{[n]_{q}}\right\|_{r}\right] \\
& \quad \leq \frac{k-1}{r}\left[\frac{(k-1)(k-2)(1+r)\left([3]_{q} r\right)^{k-2}}{[n]_{q}}+\frac{(k-1)(k-2)(1+r)\left([3]_{q} r\right)^{k-2}}{[n]_{q}}\right] \\
& \quad \leq \frac{4(k-2)(k-1)^{2}\left([3]_{q} r\right)^{k-1}}{[n]_{q}} .
\end{aligned}
$$

It follows

$$
\begin{aligned}
\left|E_{k, n}(q ; z)\right| & \leq \frac{4(k-2)(k-1)^{2}(1+r)\left([3]_{q} r\right)^{k}}{[n]_{q}^{2}}+r\left|E_{k-1, n}(q ; z)\right|+\frac{\left([3]_{q} r\right)^{k}}{[n]_{q}^{2}} D_{k} \\
& :=\frac{\left([3]_{q} r\right)^{k}}{[n]_{q}^{2}} F_{k, r}+r\left|E_{k-1, n}(q ; z)\right|,
\end{aligned}
$$


where $F_{k, r}$ is a polynomial of degree 3 in $k$ defined as $F_{k, r}=D_{k}+4(k-2)(k-1)^{2}(1+r), D_{k}$ is expressed in the above.

Since $E_{0, n}(q ; z)=E_{1, n}(q ; z)=0$ for any $z \in \mathbb{C}$, therefore, by writing the last inequality for $k=2,3, \ldots$, we easily, step by step, obtain the following:

$$
\left|E_{k, n}(q ; z)\right| \leq \frac{\left([3]_{q} r\right)^{k}}{[n]_{q}^{2}} \sum_{j=2}^{k} F_{j, r} \leq \frac{(k-1) F_{k, r}\left([3]_{q} r\right)^{k}}{[n]_{q}^{2}}
$$

As a conclusion, we have

$$
\begin{aligned}
\left|M_{n, q}(f ; z)-f(z)-\frac{z(1-z) f^{\prime \prime}(z)}{[n]_{q}}\right| & \leq \sum_{k=2}^{\infty}\left|c_{k}\right|\left|E_{k, n}(q ; z)\right| \\
& \leq \frac{1}{[n]_{q}^{2}} \sum_{k=2}^{\infty}\left|c_{k}\right|(k-1) F_{k, r}\left([3]_{q} r\right)^{k} .
\end{aligned}
$$

As $f^{(4)}(z)=\sum_{k=4}^{\infty} c_{k} k(k-1)(k-2)(k-3) z^{k-4}$ and the series is absolutely convergent in $|z| \leq[3]_{q} r$, it easily follows that $\sum_{k=4}^{\infty}\left|c_{k}\right| k(k-1)(k-2)(k-3)\left([3]_{q} r\right)^{k-4}<\infty$, which implies that $\sum_{k=2}^{\infty}\left|c_{k}\right|(k-1) F_{k, r}\left([3]_{q} r\right)^{k}<\infty$. This completes the proof of the theorem.

In the following theorem, we will obtain the exact order in approximation.

Theorem 3 Let $0<q_{n}<1$ satisfy $\lim _{n \rightarrow \infty} q_{n}=1, R>3, \mathbb{D}_{R}=\{z \in \mathbb{C} ;|z|<R\}$. Suppose that $f: \mathbb{D}_{R} \rightarrow \mathbb{C}$ is analytic in $\mathbb{D}_{R}$. Iff is not a polynomial of degree $\leq 1$, then for any $r \in\left[1, \frac{R}{3}\right)$, we have

$$
\left\|M_{n, q_{n}}(f ; \cdot)-f\right\|_{r} \geq \frac{C_{r}(f)}{[n]_{q_{n}}}, \quad n \in \mathbb{N},
$$

where $\|f\|_{r}=\max \{|f(z)| ;|z| \leq r\}$ and the constant $C_{r}(f)>0$ depends on $f, r$ and on the sequence $\left\{q_{n}\right\}_{n \in \mathbb{N}}$ but is independent of $n$.

Proof Denote $e_{1}(z)=z$ and

$$
H_{n, q_{n}}(f ; z)=M_{n, q_{n}}(f ; z)-f(z)-\frac{z(1-z) f^{\prime \prime}(z)}{[n]_{q_{n}}} .
$$

For all $z \in \mathbb{D}_{R}$ and $n \in \mathbb{N}$, we have

$$
M_{n, q_{n}}(f ; z)-f(z)=\frac{1}{[n]_{q_{n}}}\left\{z(1-z) f^{\prime \prime}(z)+\frac{1}{[n]_{q_{n}}}\left[[n]_{q_{n}}^{2} H_{n, q_{n}}(f ; z)\right]\right\} .
$$

Using the property $\|F+G\|_{r} \geq\left|\|F\|_{r}-\|G\|_{r}\right| \geq\|F\|_{r}-\|G\|_{r}$, it follows

$$
\left\|M_{n, q_{n}}(f ; \cdot)-f\right\|_{r} \geq \frac{1}{[n]_{q_{n}}}\left\{\left\|e_{1}\left(1-e_{1}\right) f^{\prime \prime}\right\|_{r}-\frac{1}{[n]_{q_{n}}}\left[[n]_{q_{n}}^{2}\left\|H_{n, q_{n}}(f ; \cdot)\right\|_{r}\right]\right\} .
$$

Considering the hypothesis that $f$ is not a polynomial of degree $\leq 1$ in $\mathbb{D}_{R}$, we get

$$
\left\|e_{1}\left(1-e_{1}\right) f^{\prime \prime}\right\|_{r}>0
$$


Indeed, supposing the contrary, it follows that $z(1-z) f^{\prime \prime}(z)=0$ for all $z \in \overline{D_{r}}$, that is $f^{\prime \prime}(z)=0$ for all $z \in \overline{D_{r}}$. Thus, $f$ is a polynomial of degree $\leq 1$, a contradiction to the hypothesis.

By Theorem 2, we get $[n]_{q_{n}}^{2}\left\|H_{n, q_{n}}(f ; \cdot)\right\|_{r} \leq M_{r}(f)$. Taking into account $\frac{1}{[n]_{q_{n}}} \rightarrow 0$ as $n \rightarrow$ $\infty$, therefore, there exists an index $n_{0}$ depending only on $f, r$ and on sequence $\left\{q_{n}\right\}_{n \in \mathbb{N}}$ such that for all $n \geq n_{0}$, we have

$$
\left\|e_{1}\left(1-e_{1}\right) f^{\prime \prime}\right\|_{r}-\frac{1}{[n]_{q_{n}}}\left[[n]_{q_{n}}^{2}\left\|H_{n, q_{n}}(f ; \cdot)\right\|_{r}\right] \geq \frac{1}{2}\left\|e_{1}\left(1-e_{1}\right) f^{\prime \prime}\right\|_{r}
$$

which implies

$$
\left\|M_{n, q_{n}}(f ; \cdot)-f\right\|_{r} \geq \frac{1}{2[n]_{q_{n}}}\left\|e_{1}\left(1-e_{1}\right) f^{\prime \prime}\right\|_{r}, \quad \forall n \geq n_{0} .
$$

On the other hand, for $n \in\left\{1,2, \ldots, n_{0}-1\right\}$, we have

$$
\left\|M_{n, q_{n}}(f ; \cdot)-f\right\|_{r} \geq \frac{W_{r, n}(f)}{[n]_{q_{n}}}
$$

where $W_{r, n}(f)=[n]_{q_{n}} \cdot\left\|M_{n, q_{n}}(f ; \cdot)-f\right\|_{r}>0$.

As a conclusion, we have

$$
\left\|M_{n, q_{n}}(f ; \cdot)-f\right\|_{r} \geq \frac{C_{r}(f)}{[n]_{q_{n}}}, \quad \forall n \in \mathbb{N}
$$

where $C_{r}(f)=\min \left\{W_{r, 1}(f), W_{r, 2}(f), \ldots, W_{r, n_{0}-1}(f), \frac{1}{2}\left\|e_{1}\left(1-e_{1}\right) f^{\prime \prime}\right\|_{r}\right\}$, this completes the proof.

Combining Theorem 3 with Theorem 1, we get the following result.

Corollary 2 Let $0<q_{n}<1$ satisfy $\lim _{n \rightarrow \infty} q_{n}=1, R>3, \mathbb{D}_{R}=\{z \in \mathbb{C}:|z|<R\}$. Suppose that $f: \mathbb{D}_{R} \rightarrow \mathbb{C}$ is analytic in $\mathbb{D}_{R}$. Iff is not a polynomial of degree 1 , then for any $r \in\left[1, \frac{R}{3}\right)$, we have

$$
\left\|M_{n, q_{n}}(f ; \cdot)-f\right\|_{r} \asymp \frac{1}{[n]_{q_{n}}}, \quad n \in \mathbb{N},
$$

where $\|f\|_{r}=\max \{|f(z)| ;|z| \leq r\}$ and the constants in the equivalence depend on $f, r$ and on the sequence $\left\{q_{n}\right\}_{n \in \mathbb{N}}$ but are independent of $n$.

Considering the derivatives of complex modified $q$-Durrmeyer type operators, we can prove the following result.

Theorem 4 Let $0<q_{n}<1$ satisfy $\lim _{n \rightarrow \infty} q_{n}=1, R>3, \mathbb{D}_{R}=\{z \in \mathbb{C}:|z|<R\}$. Suppose that $f: \mathbb{D}_{R} \rightarrow \mathbb{C}$ is analytic in $\mathbb{D}_{R}$. Also, let $1 \leq r<r_{1}<\frac{R}{3}$ and $p \in \mathbb{N}$ be fixed. If $f$ is not a polynomial of degree $\leq \max (1, p-1)$, then we have

$$
\left\|M_{n, q_{n}}^{(p)}(f ; \cdot)-f^{(p)}\right\|_{r} \asymp \frac{1}{[n]_{q_{n}}}, \quad n \in \mathbb{N},
$$


where $\|f\|_{r}=\max \{|f(z)| ;|z| \leq r\}$ and the constants in the equivalence depend on $f, r, r_{1}, p$ and on the sequence $\left\{q_{n}\right\}_{n \in \mathbb{N}}$ but are independent of $n$.

Proof Taking into account the upper estimate in Theorem 1, it remains to prove the lower estimate only.

Denoting by $\Gamma$ the circle of radius $r_{1}>r$ and center 0 , by the Cauchy's formula, it follows that for all $|z| \leq r$ and $n \in \mathbb{N}$, we have

$$
M_{n, q_{n}}^{(p)}(f ; z)-f^{(p)}(z)=\frac{p !}{2 \pi i} \int_{\Gamma} \frac{M_{n, q_{n}}(f ; v)-f(v)}{(v-z)^{p+1}} d v
$$

Keeping the notation there for $H_{n, q_{n}}(f ; z)$, for all $n \in \mathbb{N}$, we have

$$
M_{n, q_{n}}(f ; z)-f(z)=\frac{1}{[n]_{q_{n}}}\left\{z(1-z) f^{\prime \prime}(z)+\frac{1}{[n]_{q_{n}}}\left[[n]_{q_{n}}^{2} H_{n, q_{n}}(f ; z)\right]\right\} .
$$

By using Cauchy's formula, for all $v \in \Gamma$, we get

$$
M_{n, q_{n}}^{(p)}(f ; z)-f^{(p)}(z)=\frac{1}{[n]_{q_{n}}}\left\{\left[z(1-z) f^{\prime \prime}(z)\right]^{p}+\frac{1}{[n]_{q_{n}}} \frac{p !}{2 \pi i} \int_{\Gamma} \frac{[n]_{q_{n}}^{2} H_{n, q_{n}}(f ; v)}{(v-z)^{p+1}} d v\right\}
$$

Passing now to $\|\cdot\|_{r}$ and denoting $e_{1}(z)=z$, we get the following:

$$
\begin{aligned}
\left\|M_{n, q_{n}}^{(p)}(f ; \cdot)-f^{(p)}\right\|_{r} \geq & \frac{1}{[n]_{q_{n}}}\left[\left\|\left[e_{1}\left(1-e_{1}\right) f^{\prime \prime}\right]^{(p)}\right\|_{r}\right. \\
& \left.-\frac{1}{[n]_{q_{n}}}\left\|\frac{p !}{2 \pi i} \int_{\Gamma} \frac{[n]_{q_{n}}^{2} H_{n, q_{n}}(f ; v)}{(v-\cdot)^{p+1}} d v\right\|_{r}\right] .
\end{aligned}
$$

Since for any $|z| \leq r$ and $v \in \Gamma$ we have $|v-z| \geq r_{1}-r$, so, by using Theorem 2 , we get

$$
\left\|\frac{p !}{2 \pi i} \int_{\Gamma} \frac{[n]_{q_{n}}^{2} H_{n, q_{n}}(f ; v)}{(v-\cdot)^{p+1}} d v\right\|_{r} \leq \frac{p !}{2 \pi} \frac{2 \pi r_{1}[n]_{q_{n}}^{2}\left\|H_{n, q_{n}}(f ; \cdot)\right\|_{r_{1}}}{\left(r_{1}-r\right)^{p+1}} \leq \frac{M_{r_{1}}(f) p ! r_{1}}{\left(r_{1}-r\right)^{p+1}}
$$

By the hypothesis on $f$, we have

$$
\left\|\left[e_{1}\left(1-e_{1}\right) f^{\prime \prime}\right]^{(p)}\right\|_{r}>0
$$

Indeed, supposing the contrary, it follows that $\left\|\left[e_{1}\left(1-e_{1}\right) f^{\prime \prime}\right]^{(p)}\right\|_{r}=0$, that is, $z(1-z) f^{\prime \prime}(z)$ is a polynomial of degree $\leq p-1$. Let $p=1$ and $p=2$, then the analyticity of $f$ obviously implies that $f$ is a polynomial of degree $\leq 1=\max (1, p-1)$, a contradiction.

Now let $p \geq 3$, then the analyticity of $f$ obviously implies that $f$ is a polynomial of degree $\leq p-1=\max (1, p-1)$, a contradiction to the hypothesis.

In conclusion, $\left\|\left[e_{1}\left(1-e_{1}\right) f^{\prime \prime}\right]^{(p)}\right\|_{r}>0$, and in continuation reasoning exactly as in the proof of Theorem 3, we can get the desired conclusion.

Remark 4 If we use King's approach to consider a King-type modification of the complex extension of the operators which was given by (1.1), we will obtain better approximation (cf. [15-17]). 


\section{Competing interests}

The authors declare that they have no competing interests.

\section{Authors' contributions}

All authors contributed equally and significantly in writing this article. All authors read and approved the final manuscript.

\section{Acknowledgements}

This work is supported by the National Natural Science Foundation of China (Grant No. 61170324), the Natural Science Foundation of Fujian Province of China (Grant No. 2010J01012), the Class A Science and Technology Project of Education Department of Fujian Province of China (Grant No. JA12324), and the National Defense Basic Scientific Research Program of China (Grant No. B1420110155).

Received: 25 March 2012 Accepted: 7 September 2012 Published: 2 October 2012

\section{References}

1. Kac, VG, Cheung, P: Quantum Calculus. Universitext. Springer, New York (2002)

2. Gasper, G, Rahman, M: Basic Hypergeometric Series. Encyclopedia of Mathematics and Its Applications, vol. 35. Cambridge University Press, Cambridge (1990)

3. Philips, GM: Bernstein polynomials based on the q-integers. Ann. Numer. Math. 4, 511-518 (1997)

4. Agratini, O, Nowak, G: On a generalization of Bleimann, Butzer and Hahn operators based on q-integers. Math. Comput. Model. 53(5-6), 699-706 (2011)

5. Aral, A: A generalization of Szász-Mirakyan operators based on q-integers. Math. Comput. Model. 47, 1052-1062 (2008)

6. Doğru, O, Orkcu, M: Statistical approximation by a modification of q-Meyer-König-Zeller operators. Appl. Math. Lett. 23, 261-266 (2010)

7. Gupta, V, Radu, C: Statistical approximation properties of $q$-Baskakov Kantorovich operators. Cent. Eur. J. Math. 7(4)، 809-818 (2009)

8. Gal, SG: Voronovskaja's theorem, shape preserving properties and iterations for complex $q$-Bernstein polynomials. Studia Sci. Math. Hung. 48(1), 23-43 (2011)

9. Mahmudov, NI: Approximation properties of complex q-Szász-Mirakjan operators in compact disks. Comput. Math. Appl. 60,1784-1791 (2010)

10. Mahmudov, NI: Approximation by genuine $q$-Bernstein-Durrmeyer polynomials in compact disks. Hacet. J. Math. Stat. 40(1), 77-89 (2011)

11. Ostrovska, S: q-Bernstein polynomials of the Cauchy kernel. Appl. Math. Comput. 198, 261-270 (2008)

12. Gupta, $\mathrm{V}$, Wang, $\mathrm{H}$ : The rate of convergence of $q$-Durrmeyer operators for $0<q<1$. Math. Methods Appl. Sci. 31 , 1946-1955 (2008)

13. Agarwal, RP, Gupta, V: On q-analogue of a complex summation-integral type operators in compact disks. J. Inequal. Appl. 2012(1), 111 (2012). doi:10.1186/1029-242X-2012-111

14. Videnskii, VS: On $q$-Bernstein polynomials and related positive linear operators. In: Problems of Modern Mathematics and Mathematical Education, pp.118-126. Hertzen Readings, St. Petersburg (2004) (in Russian)

15. King, JP: Positive linear operators which preserve $x^{2}$. Acta Math. Hung. 99(3), 203-208 (2003)

16. Mahmudov, Nl: q-Szász-Mirakjan operators which preserve $x^{2}$. J. Comput. Appl. Math. 235, 4621-4628 (2011)

17. Doğru, O, Örkcü, M: King type modification of Meyer-König and Zeller operators based on the q-integers. Math. Comput. Model. 50, 1245-1251 (2009)

doi:10.1186/1029-242X-2012-212

Cite this article as: Ren and Zeng: Approximation by a kind of complex modified $q$-Durrmeyer type operators in compact disks. Journal of Inequalities and Applications 2012 2012:212.

\section{Submit your manuscript to a SpringerOpen ${ }^{\ominus}$ journal and benefit from:}

- Convenient online submission

- Rigorous peer review

Immediate publication on acceptance

- Open access: articles freely available online

- High visibility within the field

- Retaining the copyright to your article 